BIOLOGICAL CHEMISTRY

\section{Catalytic competition for cells}

\author{
Virginia W. Cornish
}

\section{Ways of evolving proteins, and assessing the vast numbers of variants needed to identify those with novel enzymatic activity, are themselves evolving. Oil droplets containing basic cell machinery provide a promising approach.}

The cell has the enviable ability to evolve through mutation of its hereditary DNA code. When we first learned how to mutate DNA in the test tube, and so manipulate the aminoacid sequence of a protein, we quickly learned how difficult it is to rationally alter protein function with just a few amino-acid changes ${ }^{1}$. However, modern DNA technology makes it possible to generate not one or two but $10^{10}$ or more protein variants with an altered aminoacid sequence, and we can now carry out 'directed evolution' - of, for example, surrogates of green fluorescent protein that range in colour from cyan to red ${ }^{2}$. But what will it take to compete with the evolutionary power of the cell to create and identify even more dramatic changes in function?

As described in back-to-back papers published in Chemistry \& Biology, groups led by Dan Tawfik ${ }^{3}$ and Andrew Griffiths ${ }^{4}$ attempt to extend the directed evolution of enzyme catalysis to chemistry beyond that naturally carried out in the cell. Proteins with new functions presumably evolve in the cell through the accumulation of mutations in genomic DNA generated by random genetic drift, followed by selective amplification of cells with the fittest variants when some selective pressure is applied. Directed evolution seeks to recapitulate this process on an experimentally accessible timescale by selectively introducing mutations into the DNA encoding the protein of interest at a high rate, and then picking the handful of protein variants that have acquired the desired new function ${ }^{5}$.

Synthesizing $10^{10}$ protein variants at the DNA level is, in fact, easy. Variations of the polymerase chain reaction, a technique for selectively amplifying a segment of DNA, make it possible not only to mutate select amino acids in the active site of a protein, but also to replace a whole loop in a protein or mimic natural recombination by swapping whole segments from a related protein sequence. (Note, however, that $10^{10}$ sequence variants is a tiny number compared with all the possible sequence variants for even a 200 -amino-acid protein composed of 20 different amino-acid building-blocks.)

But with such large numbers, identifying the handful of proteins with the desired new function is very hard. The cell offers an elegant solution to this problem of finding the needle in the haystack. It acts as a self-replicating compartment that links a mutatable and amplifiable DNA code to the catalyst
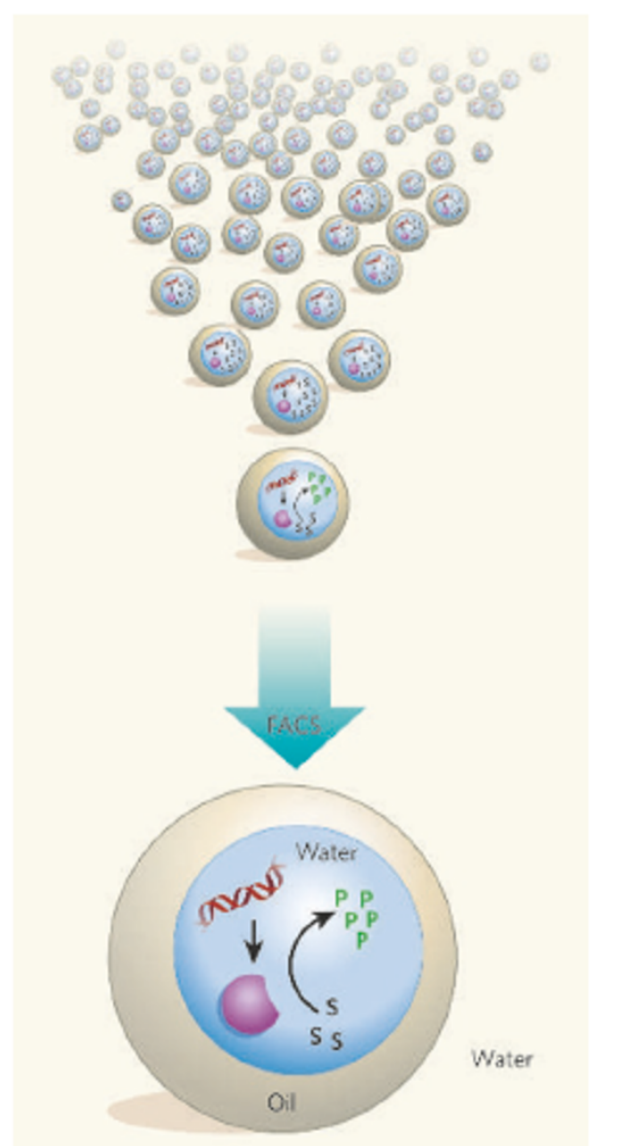

Figure 1 | Finding the needle in the haystack ${ }^{34}$. Water-in-oil-in-water emulsions of the cells protein-synthesis machinery allow fluorescenceactivated 'cell' sorting (FACS) of individual droplets containing not only a protein and its unique DNA sequence, but also fluorogenic reporters for different chemical transformations. Individual droplets that contain enzyme variants (purple) with increased catalytic activity can be sorted at a rate of about $10^{7}$ per hour based on the number of fluorescent product molecules (P) synthesized from the substrate (S). The DNA (red) from active droplets can then be recovered and amplified.

translated from that DNA, and to the myriad functions that determine the fitness of the cell. Some methods (phage display, for example) can directly or indirectly link each protein physically to its unique DNA sequence, even for $10^{10}$ variants, and have been exploited for the de novo evolution of binding proteins ${ }^{6}$. But they do not lend themselves readily to high-throughput assays for enzyme catalysis.
Traditional enzyme assays can be carried out one-by-one in microtitre plates using automation techniques. But signal-to-noise issues limit these assays to smaller numbers of protein variants in practice. For the vast range of chemical transformations not carried out in the cell, we do not have the assays to sort through the large number of protein variants, and the directed evolution of de novo catalysts eludes us.

Tawfik, Griffiths and their co-workers have confronted this problem by developing an 'in vitro compartmentalization' (IVC) technology that basically strips the cell's machinery for transcribing and translating DNA to RNA to protein, and reconstitutes it in water-in-oil droplets that have about the same volume as a bacterial cell. This approach provides one solution for linking each unique protein variant to its DNA sequence, because statistically it is easy to create $10^{10}$ water-in-oil droplets each containing a unique DNA sequence encoding a unique protein variant.

Tawfik and Griffiths have already successfully used their IVC technology for test-tube evolution of proteins, but largely for enzymes involved in modifying DNA. In the new papers $^{3.4}$ they go further, providing not only a link between the DNA and the protein it encodes, but also a functional assay that can handle large numbers of variants.

Both groups show that they can make waterin-oil-in-water emulsions that allow the encapsulation of fluorogenic indicator dyes used to detect enzyme catalysis in more traditional formats. They then submit some $10^{7}$ droplets, each containing a unique protein variant, to a technique known as fluorescence-activated 'cell' sorting (FACS; Fig. 1). Using FACS on these water-in-oil-in-water emulsions, they can carry out test-tube evolution to increase the catalytic activity of a known protein.

Tawfik and co-workers ${ }^{3}$ leave behind the IVC technology and literally encapsulate abacterial cell. They show that this increases the concentration of enzyme that can be produced in each individual droplet to about $10^{5} \mathrm{~mol}-$ ecules. From just one round of mutation and FACS screening, they then isolate a variant of the natural enzyme paraoxonase with a 100 -fold increase in hydrolytic activity from $10^{6}$ different variants. Griffiths and co-workers ${ }^{4}$ carry out FACS with cell-free IVC droplets, which synthesize about 100 copies of protein per droplet. Returning to a classic experiment in directed evolution, they evolve $\mathrm{Ebg}$, a protein of unknown function made by the bacterium Escherichia coli, into a $\beta$-galactosidase enzyme using a fluorogenic $\beta$-galactosidase substrate. With multiple rounds of mutation and screening, they isolate several Ebg variants showing a more than 300 -fold increase in $\beta$-galactosidase activity compared with Ebg.

So what is the best way to compete with the evolutionary power of the cell? Tawfik and Griffiths strip the cell of some of its basic machinery, and, by analogy to the cell, 
compartmentalize this machinery in a waterin-oil droplet. Their IVC system preserves the cell's means of linking DNA to protein, and then adds on in vitro chemistry to create the evolutionary pressure. At the other extremes are completely synthetic encoded system $\mathrm{s}^{7-9}$, or solutions that seek to expand the chemistry carried out by the cell ${ }^{10}$. The advantage of a completely synthetic system may be that it can go beyond the chemistry that can be synthesized or tolerated by the cell, although the range of chemistry naturally carried out by the cell is awfully impressive.

The field of directed evolution is in a vibrant phase $\mathrm{e}^{11,12}$. Beyond that, this tinkering with cells will provide useful technologies for genomics and biomedical research, and will inspire thinking about what might be synthesized to recapitulate the functions of the cell and how the cell might be co-opted for new functions ${ }^{13,14}$.

Virginia W. Cornishis in the Department of Chemistry, Columbia University, Havemeyer Hall, MC 3111, 3000Broadway, New York, New York 10027, USA.

e-mail:vc114@columbia.edu

1. Knowles, J.R. Science 236, 1252-1258 (1987).

2. Shane, N. C, Steinbach, P. A.\& Tsien, R. Y. Nature Meth 2 , 905-909 (2005).

3. Aharoni, A, Amitai, G, Bernath, K, Magdassi, S. \& Tawfik, D. S. Chem. Biol. 12, 1281-1289 (2005)
4. Mastrobattista, E etal. Chem. Biol. 12, 1291-1300 (2005).

5. Amold F. H. Nature 409, 253-257 (2001).

6. Birz, H. K, Amstutuz, P. \&.Pluckthun A.Nature Biatechnal. $23,1257-1268$ (2005)

7. Sculimbrene, B. R.\& Miller, S. I. J.Am Chem. Soc. 123, 10125-10126(2001)

8. Gartner, ZJ.etal. Sdence 305, 1601-1605 (2004)

9. Halpin, D.R \& Harbury, P. B. PLOS Biol. 2, e174 (2004).

10. Lin, H, Tao, H.\& Cornish, V.W.J. Am. Chem Soc 126, 15051-15059 (2004)

11. Yoshilkuni, Y, Ferrin, T.E.\& Keasling L. D. Nature advance online publication do:101088/nature 04607 (2006).

12. Park.H.S.et al. Science 311,535-538 (2006)

13. Szostak, I.W, Bartel, D.P.\& Luisi, P. L. Nature 409, $387-390$ (2001)

14. Bennec, S. A. \& Sismour, M. Nature Rex Genet. 6, 533-543 (2005).

\section{MOLECULAR PHYSICS}

\section{Recombination cool and fast}

\section{Benjamin J. McCall}

\section{Molecular physicists and astrophysicists alike would like to know how fast the $\mathrm{H}_{3}^{+}$molecular ion recombines with electrons. Fast, seems to be the answer - with an awkward consequence for the astrophysicists.}

Every schoolchild knows that, like opposing poles of a magnet, opposite charges attract. But what happens when charged bodies are small enough that the rules of quantum mechanics come to bear, for instance when an electron and a positively charged molecule attract? Here, the situation is more complicated: even the reaction between an electron and the simplest polyatomic molecule, $\mathrm{H}_{3}^{+}$(which can be thought of as a hydrogen molecule, $\mathrm{H}_{2}$, with an extra proton, $\mathrm{H}^{+}$) has puzzled both theorists and experimentalists for decades. In a contribution to Physical Review Letters, Kreckel et al. ${ }^{1}$ describe an ingenious experiment that provides further elucidation of the speed of this fundamental reaction.

When an electron approaches a singly charged positive ion (call it $\mathrm{X}^{+}$), both bodies experience an attraction that accelerates them and causes them to collide. They can recombine to form neutral $\mathrm{X}$, provided that the extra kinetic energy that they have gained by being accelerated can somehow be removed. For macroscopic objects, this is not generally a problem: friction dissipates the energy. At the quantum-mechanical level, however, this cannot happen. If $\mathrm{X}^{+}$is the ion of a single atom, energy can be lost only by emitting a photon, a slow process that seldom happens during the short time a collision takes. In most such collisions, the ion and electron fly away from each other again. If $\mathrm{X}^{+}$is a molecular ion, however, there is a much more efficient option: the molecule can break apart following recombination with the electron, and the resulting neutral fragments can carry away kinetic energy. This is the process known as dissociative recombination.

The $\mathrm{H}_{3}^{+}$ion assumes an important role in astrophysics as the first link in a chain of chemical reactions in interstellar clouds through which most of the molecules found in interstellar space form (Fig. 1). Interstellar clouds were recently seen to contain much more $\mathrm{H}_{3}^{+}$than expected ${ }^{2}$, bringing the persistent enigma of its recombination rate back to the fore. Exactly how the dissociative recombination of $\mathrm{H}_{3}^{+}$works was explained theoretically only recently ${ }^{3,4}$, and, starting in 1973 , many experimental measurements have yielded drastically differing values for the rate at which it occurs 5 .

$\mathrm{H}_{3}^{+}$is produced in ionized gases known as plasmas. Different plasma conditions will lead to different degrees of vibrational and rotational excitation of the $\mathrm{H}_{3}^{+}$ions, perhaps accounting for some of the variation in the experimental recombination rates. If the rate of recombination were lower than assumed, especially at the lower temperatures of interstellar space, the overabundance of $\mathrm{H}_{3}^{+}$in diffuse clouds could be easily explained. But without accurate values for the rate, further progress in understanding the mystery of $\mathrm{H}_{3}^{+}$abundance is impossible.

Hence the efforts to understand dissociative recombination in terrestrial laboratories. In order to best simulate the conditions in

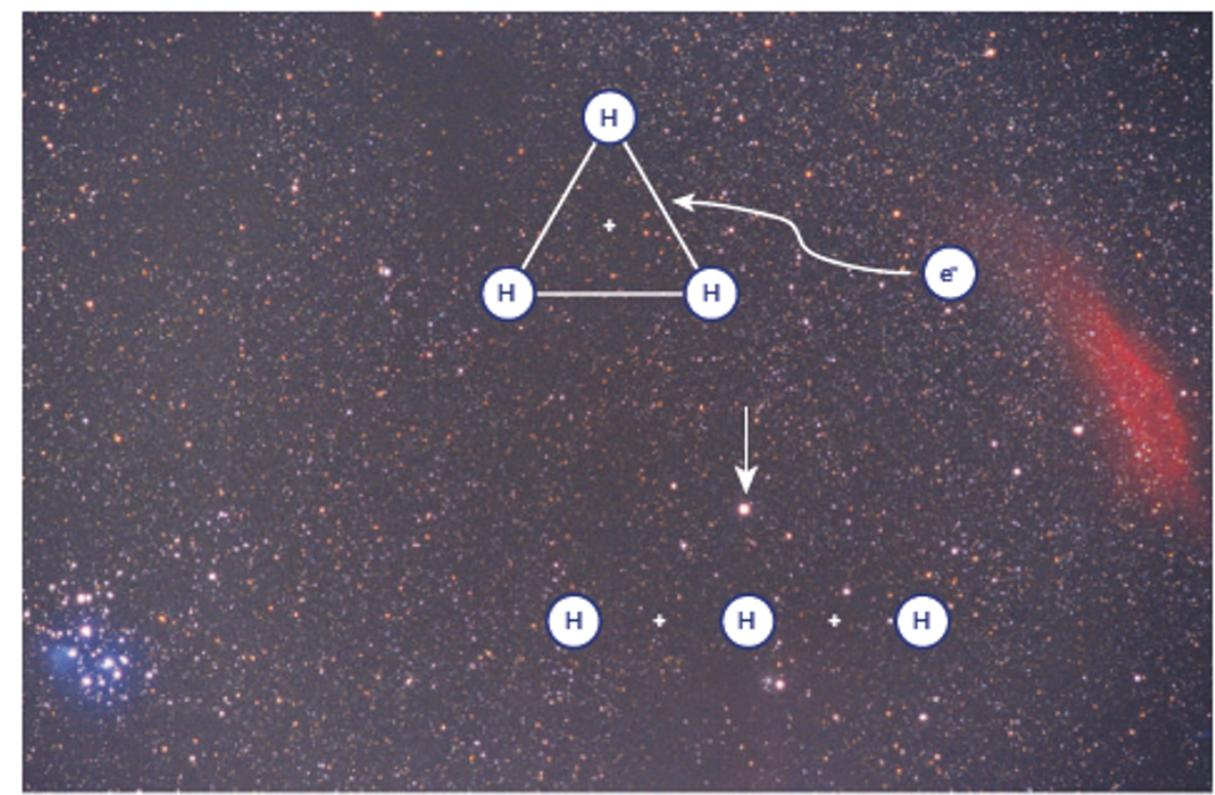

Figure 1 | Cosmicmystery. When an electron and a positively charged $\mathrm{H}_{3}^{+}$ion meet, three separate neutral hydrogen atoms are formed in a process known as dissociative recombination. The image is overlaid on a wide-field image of the Perseus region: the bright stars at bottom left are the Pleiades, or Seven Sisters, and the red region to the right is the California nebula. The bright star at the arrow's head is $\zeta$ Persei, where $\mathrm{H}_{3}^{+}$has been observed in unexpectedly high abundance. ${ }^{2}$. The results of Kreckel et al. ${ }^{1}$ imply this cannot be due to slow dissociative recombination. The most likely explanation is instead an enhanced rate of ionization by cosmic rays. 